\title{
Optimizing expectations to prevent side effects and enhance quality of life in breast cancer patients undergoing endocrine therapy: study protocol of a randomized controlled trial
}

\author{
Pia von Blanckenburg ${ }^{1}$, Franziska Schuricht ${ }^{1}$, Ute-Susann Albert ${ }^{2}$, Winfried Rief ${ }^{1}$ and Yvonne Nestoriuc $^{3 *}$
}

\begin{abstract}
Background: Adjuvant endocrine therapy can improve disease-free survival and time before recurrence in breast cancer patients. However, it is associated with considerable side effects that negatively affect patients' quality of life and cause non-adherence. The recently demonstrated effect of individual expectations on side-effect development (nocebo effect) suggests that psychological factors play a role in the prevention of side effects. The aim of this study is to evaluate cognitive-behavioral side-effect prevention training (SEPT) for breast cancer patients. This article describes the study protocol and applied research methods.
\end{abstract}

Methods/Design: In a randomized controlled trial, 184 female breast cancer patients are assigned to receive either SEPT, standard medical care or a manualized supportive therapy at the start of adjuvant endocrine treatment. SEPT consists of three sessions of cognitive-behavioral training including psychoeducation to provide a realistic view of endocrine therapy, imagination-training to integrate positive aspects of medication into daily life, and side-effect management to enhance expectations about coping ability. Side effects three months after the start of endocrine therapy serve as primary outcomes. Secondary outcomes include quality of life, coping ability and patients' medication adherence. Patients' expectations (i.e., expectations about side effects, coping ability, treatment and illness) are analyzed as mediators.

Discussion: The optimization of expectations might be a potential pathway in health care to improve patients' quality of life during long-term medication intake. The results will provide implications for a possible integration of evidence-based prevention training into clinical practice.

Trial registration: ClinicalTrials.gov, (NCT01741883).

Keywords: Nocebo, Side effects, Prevention, Breast cancer, Endocrine therapy, RCT

\section{Background}

Endocrine therapy is the pivotal adjuvant treatment for over $75 \%$ of breast cancer patients. National and international guidelines recommend it for long-term intake, over at least five years, in patients with hormone-receptor-positive primary breast cancer [1,2]. Adjuvant endocrine therapy reduces the risk of cancer recurrence, development of metastases, and cancer mortality [3,4]. Despite these benefits, almost every second patient

\footnotetext{
*Correspondence: yvonne.nestoriuc@uni-hamburg.de

${ }^{3}$ Department of Clinical Psychology, Hamburg University, Hamburg, Germany Full list of author information is available at the end of the article
}

discontinues treatment [5], while another $17 \%$ refuse to initiate drug intake [6]. Reported non-adherence rates range from $35 \%$ to $50 \%[7,8]$, leading to increased mortality in women with breast cancer [9]. Main reasons for non-adherence are side effects that reduce patients' quality of life [10]. Thus, effective side-effect management and side-effect prevention are crucial.

Most frequently reported side effects of endocrine therapy are arthralgia, hot flushes, weight gain, mood swings, loss of libido and vaginal dryness $[11,12]$. To a great extent these symptoms are directly caused by the pharmacodynamics of the treatment, depleting women

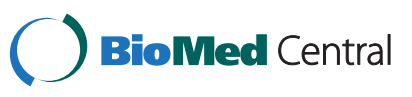


of female sex hormones, thereby initiating or adding up to typical menopausal discomforts. In contrast, longterm evaluations of adjuvant hormonal therapy showed that a substantial proportion of the reported side effects were not related to the treatment [13]. These side effects, such as headaches, skin irritation, dizziness, nausea and gastrointestinal irritation, have no known pharmacological association with endocrine treatment and are therefore termed non-specific medication side effects [14]. They are influenced by patient characteristics such as expectations and pre-existing symptoms. It is assumed that analog processes are involved in the nocebo (Latin: "I shall harm") phenomenon, in which placebo-pills cause adverse effects [15]. Hence, a randomized placebo-controlled study of letrozole showed that around 20\% of breast cancer patients in the placebo arm experienced typical menopausal symptoms [16]. Thus, negative expectations can not only influence the occurrence of nonspecific side effects but even worsen specific side effects via the nocebo-mechanism (nocebo effect) $[17,18]$.

Negative expectations about medication have been shown to predict the incidence of non-specific side effects in patients with rheumatoid arthritis, even if disease severity and medication regimen were controlled [19]. Expectations about occurrence and intensity of side effects seem to be an important predictor of side effects in cancer treatment [20], for example chemotherapyrelated nausea [21]. A recent meta-analysis showed a significant, medium-sized association between patients' expectations of side effects and the actual experience of these side effects from cancer treatments [22]. In a pilot study by our group, response expectations predicted the incidence of side effects three months after the start of endocrine treatment [23,24]. Furthermore, cognitive representations and expectations about the consequences of illness were found to be associated with physical health outcomes in breast cancer patients [25]. Leventhal's self-regulation model of health [26] additionally focuses on expected coping with illness [27] and the emotional representations of an illness [26]. Thus, anxiety [28] and depression may be relevant in the development of nocebo effects and non-specific side effects [14]. Several other factors appear to be of importance in this context: e.g., prior experiences with side effects [22], higher pre-existing symptoms [29], and the tendency toward somatization, symptom amplification and selective attention on bodily sensations [14], all of which can result in a possible misinterpretation of prior existing symptoms as side effects of the cancer medication [30]. Taken together, patients' expectations seem to be essential for the development of side effects. Optimizing these expectations might be a promising way to minimize patients' side-effect burden during long-term intake of endocrine medication.
Only few studies have tried to optimize expectations in cancer treatment. Changes in illness representations are associated with less fear of progression [31], and patients with high expectations for treatment-induced nausea could profit from a positive expectation-manipulation [32]. In particular, the way information about side effects is given to the patients is crucial for the development of expectations. It is recommended that information be framed in a positive way, e.g., not only explain possible side effects but also the expected benefits of the medication. Further, it is important to promote a positive doctorpatient interaction and to foster effective management of symptoms [33]. Recent studies showed that cognitive behavioral therapy helps breast cancer patients in the management of menopausal side effects and can lead to decreased levels of reported symptoms [34,35]. So far, no study has focused on optimizing expectations to prevent side effects. We therefore developed a psychological sideeffect prevention training (SEPT) to prevent side effects by optimizing patients' expectations, which may be an effective pathway to enhance overall quality of life. This article describes the study design and the research methods to answer the following research questions:

1. Is a three-session psychological intervention effective in reducing side effects and improving quality of life during long-term intake of endocrine therapy?

2. Do treatment and side-effect expectations mediate the beneficial effects?

3. Are there certain patient characteristics that predict which patients benefit the most from the training?

\section{Methods/Design}

\section{Study design}

The study is designed as dual-center, randomized controlled trial with three arms and follow-up assessment (see Figure 1). The study procedure is implemented at the Department of Gynecology, Gynecological Endocrinology and Oncology, Philipps University Marburg, Germany, and at the University Medical Center Hamburg-Eppendorf, Germany. Participants are women with hormone-receptor-positive breast cancer, scheduled to start adjuvant endocrine treatment. After signing informed consent to participate in the study and receiving medical information about endocrine treatment from the hospital staff, all patients are provided additional standardized information and patient education about the scheduled treatment by a trained research assistant/ clinical psychologist to homogenize knowledge about endocrine treatment. The structured treatment information is given verbally and through a leaflet, illustrating the physical mode of action, the desired effects, and the potential side effects of endocrine therapy. After completing baseline assessment (including symptom status and pre-treatment side-effect 


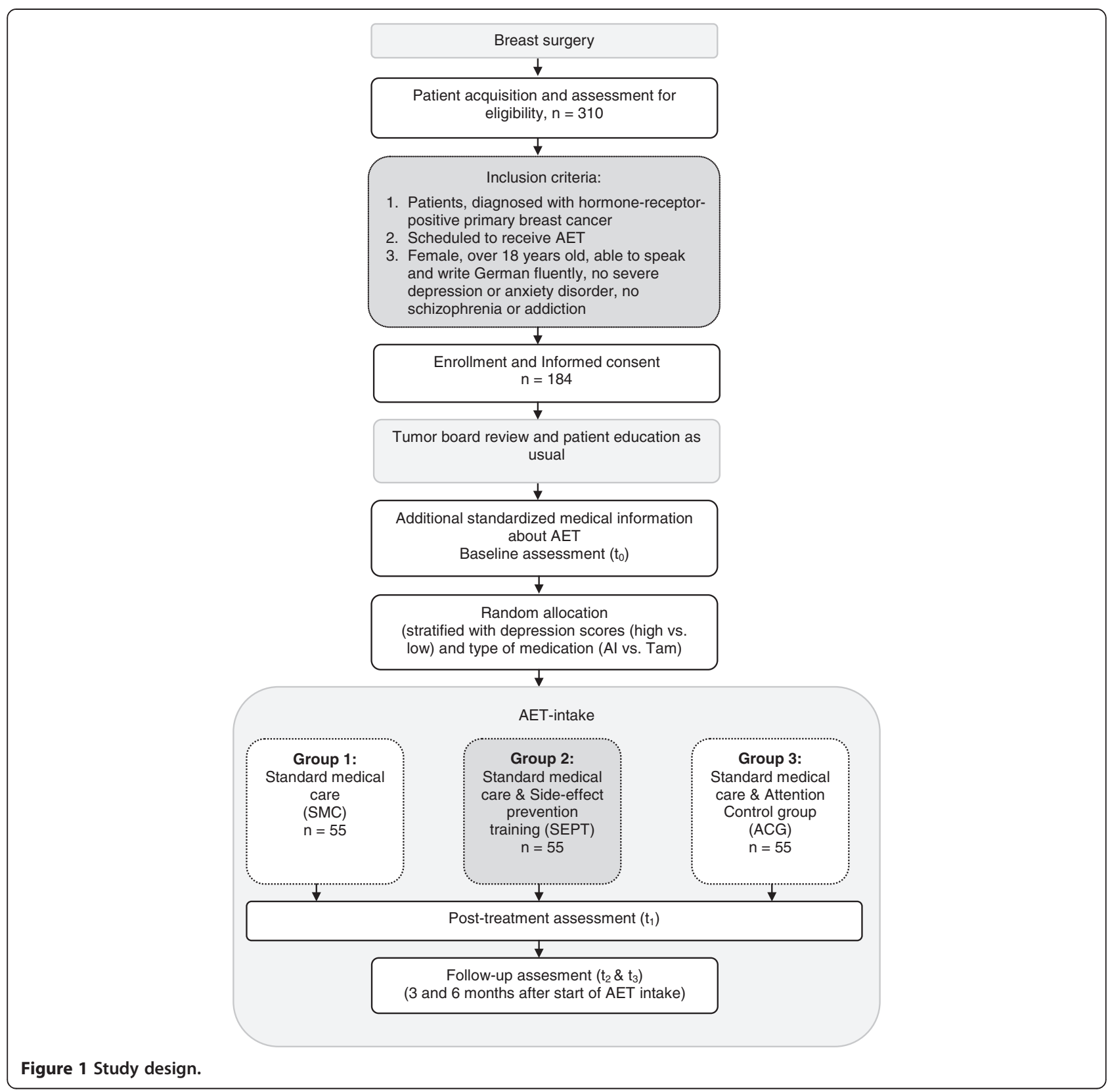

expectations) patients are randomly assigned to one of three groups. Group 1 receives standard medical care (SMC) only. Group 2 receives SMC and the side-effect prevention training (SEPT). Group 3 receives SMC and supportive therapy as an attention control group (ACG). Outcomes are assessed homogeneously in all three groups, three and six months after the start of medication intake.

\section{Participants}

Patients with hormone-receptor-positive primary breast cancer scheduled to undergo first-line endocrine treatment with tamoxifen (and additional GnRH-analoga, depending on the menopausal status) or a third-generation aromatase inhibitor (i.e., anastrozole, letrozole or exemestan) are eligible. Exclusion criteria are the presence of a serious co-morbid psychiatric condition (schizophrenia, addiction, severe affective or severe anxiety disorder), the presence of a life-threatening co-morbid (non-cancer) medical condition, insufficient German language skills, and cognitive inability to give informed consent. Inclusion and exclusion criteria are checked by screening patients' history and medical records and standardized assessment using the structured psychiatric interview "mini-dips" [36]. 


\section{Recruitment and informed consent}

Diagnosis and treatment are initiated independently of the study according to the German breast cancer guidelines by the interdisciplinary tumor board of the breast cancer center. Patients are required to have completed primary surgery and/or chemotherapy, if indicated.

Patients are screened for eligibility during their hospital stay in the breast cancer center. Women meeting the inclusion criteria are informed about the study concept and invited by their oncologists to take part in the study. Patients who give written informed consent after receiving detailed written and verbal information are admitted to the study. Participation is voluntary and can be withdrawn by the patient any time with no disadvantages.

\section{Ethics}

The study protocol was approved by the medical ethics committee of the Philipps University Marburg, and the Hamburg Medical Chamber. The study will be conducted in accordance with the Declaration of Helsinki, Good Clinical Practice guidelines, including data and patients' privacy protection.

\section{Sample size calculation}

Required sample sizes were determined a priori with G-Power [37]: The sample size for the randomized group comparison was determined according to a MANOVA with repeated measures, testing for a three groups $\mathrm{x}$ three time points interaction. Assuming a medium effect size of $f(V)=.20, N=153$ patients ( $=51$ per group) are needed to achieve $80 \%$ power.

Taking into account a potential drop-out rate of $20 \%$ (e.g., due to exhaustion or medical complications), we aim to include a total of 184 breast cancer patients.

\section{Quality standards (Minimization of bias) \\ Randomization and blinding}

Randomization follows after completing baseline assessment. Patients are equally allocated to one of the three treatment arms using sealed envelopes. Assignment follows a stratified permuted block randomization procedure with a block size of nine. Stratification criteria $(2 \times 2)$ are the sum score ( $\leq 13$ vs. $>13)$ of the hospital anxiety and depression questionnaire (HADS-D) $[38,39]$ during hospital stay and type of medication (aromatase inhibitor vs. tamoxifen). Assignment sequence is generated by staff who are not involved in the intervention process and conducted electronically using the statistical program WINPEPI [40]. The research assistants responsible for the assessment are blinded to group allocation.

\section{Attrition bias}

In order to examine potential attrition bias, drop-out analyses will be performed. In accordance with the intentionto-treat principle, the data of all patients randomised to the treatment groups will be analysed.

\section{Control for therapeutic allegiance}

To ensure the comparability of treatments, treatment dose, application and assessment occasions for SEPT and ACG will be identical.

All therapists are clinical psychologists with advanced cognitive behavioral training and have comparable professional experience. They are trained in the use of the treatment manual before the trial starts. Training includes a full treatment cycle with at least one patient per treatment group using video feedback and professional supervision of each treatment session. During the whole study therapists are under ongoing supervision by highly experienced psycho-oncologists. Both types of interventions are manualized and each therapist will treat a comparable number of patients in each group. Treatment fidelity is assessed and rated before approval of therapists to start in this trial. All sessions will be videotaped and an amount of $33 \%$ will be selected randomly and rated by an independent rater.

\section{Psychological interventions}

In the intervention groups three individual sessions of 50-75 minutes are being held with a clinical psychologist over the course of three to four weeks (see Figure 2).

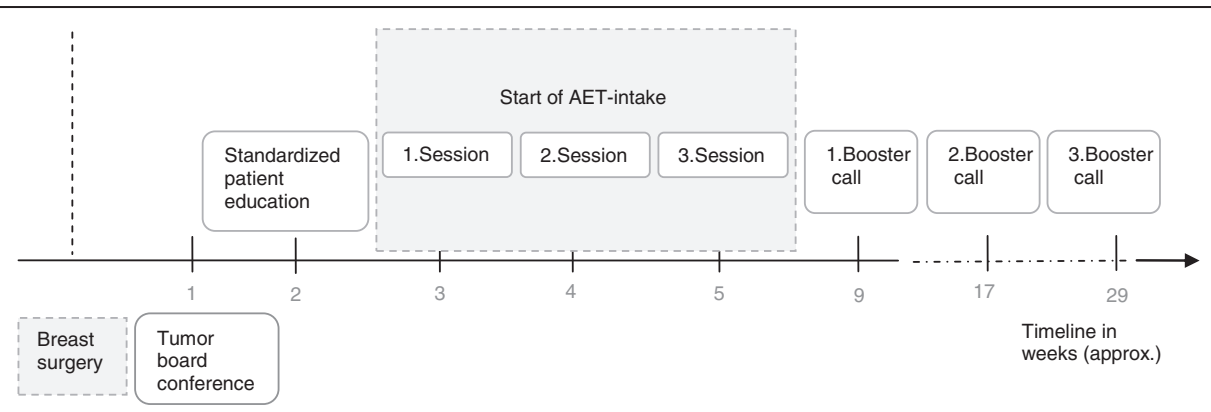

Figure 2 Intervention schedule. 
One, three and six months after the intervention booster telephone calls will be made in both intervention groups.

\section{Intervention: side-effect prevention training}

The goal of SEPT is the prevention of non-specific and nocebo side effects from endocrine therapy by optimizing treatment- and illness-related expectations. Contents of the training are psychoeducation [41] to provide a realistic view of the treatment, imagination-training to integrate positive aspects of medication into daily life and side-effect management [42] to enhance expectations about individual coping abilities. All intervention components and goals are listed in Table 1. SEPT is a manual-based program. Individual topics are adapted specifically to each patient according to her individual expectations. The baseline questionnaires are used as a starting point for tailoring the intervention so it is consistent with the written treatment guidelines. Patients in the SEPT group receive a booklet with patient material detailing the contents, goals and interventions of each session. The booklet also contains work sheets, postcards and further material that can be personalized by each participant. Sessions will focus on subsequent topics:

Session 1: At the beginning of the first session, psychoeducation about the active principle of endocrine treatment is given, tailored to the individual patient's needs. The adverse side effects most expected by the patient are discussed and contrasted with treatment benefits.

A guided imagination is performed to visualize the positive aspects (e.g., protection from cancer recurrence) of the endocrine treatment. The imagination is recorded on audio file and incorporated into patients' daily handling of the medication. Furthermore, the impact of expectations on side effects (e.g., pain following negative expectations but without the administration of any inert substance) is discussed and the concept "nocebo phenomenon" explained to the patients.

Session 2: Session two focuses on the development of coping strategies for side-effect management that are implemented in a written problem-solving scheme for the three most expected or dreaded side effects. Strategies employed include behavioral techniques, cognitive strategies, dietary advice, physical exercises, identification and avoidance of triggers for specific side effects. Patients are asked to create a "tool-box" at home filled with useful material to implement the discussed copingstrategies.

Session 3: This last session includes skills training for improved patient-physician communication to positively influence expectations about breast cancer check-ups. Additionally, the role of attention for the development of non-specific side effects and the worsening of specific side effects is discussed. Patients are encouraged to re
Table 1 Intervention components and goals of the side-effect prevention training

\begin{tabular}{|c|c|}
\hline Intervention components & Goals \\
\hline \multicolumn{2}{|l|}{ Session 1} \\
\hline - Psychoeducation about AET & $\begin{array}{l}\text { - Knowledge about AET and } \\
\text { nocebo effect }\end{array}$ \\
\hline $\begin{array}{l}\text { - Guided imagination and visualization } \\
\text { of positive treatment aspects }\end{array}$ & $\begin{array}{l}\text { - Strengthen control and } \\
\text { benefit expectations }\end{array}$ \\
\hline $\begin{array}{l}\text { - Psychoeducation about nocebo and } \\
\text { non-specific side effects }\end{array}$ & $\begin{array}{l}\text { - Integration of positive aspects } \\
\text { of AET into daily routines }\end{array}$ \\
\hline
\end{tabular}

\section{Homework}

- Practice relaxation and imagination (anchored by CD)

- Further creative work with

imagination, e.g., painting

\section{Session 2}

- Develop individual problem-solving scheme for the three most important side effects

- Create an action plan for behavioral and cognitive strategies

\section{Homework}

- Complete and modify the personal problem-solving scheme

- Create an individual "tool box"

- Practice relaxation and imagination (anchored by CD)

\section{Session 3}

- Psychoeducation about doctorpatient communication

- Develop distraction strategies of for the time of AET intake

Optimizing coping expectations

- Improving patient-physician communication

- Summing up and outline treatment goals

\section{Homework}

- Practice relaxation and imagination (anchored by CD)

- Complete and modify the personal problem-solving scheme and the tool box

\section{Telefone booster calls}

\begin{tabular}{l} 
- Compare expected and occurred $\quad \begin{array}{l}\text { - Maintain optimized } \\
\text { side expectations }\end{array}$ \\
- Check practicability of the coping \\
strategies and the "tool box" and \\
modify if necessary \\
\hline Notes: $A E T$ = Adjuvant endocrine therapy
\end{tabular}

(activate) individual resources and activities helping to distract from the potential occurence of side effects, but also strenghten the patient for the time of medication intake. At the end of the session, all topics of the previous sessions are reviewed. 
Booster calls: The three phone calls focus on repeating the contents of the intervention. The therapeutic contact is used to give support to the patients during the first time of medication intake. Many patients describe it as very helpful to talk about their concerns in this phase of illness and treatment (the phase of rehabilitation after being discharged from hospital). During the booster calls, possible problems with the relaxation and imagination training are discussed and resolved whenever possible. The coping strategies are reviewed and adapted. Furthermore, the problem-solving scheme will be extended to further side effects including new coping strategies.

\section{Attention control group (ACG): supportive therapy}

Supportive therapy serves as an attention control group for non-specific factors such as therapist's attention and patient-therapist relationship. It serves to distinguish specific effects of SEPT from psychological placebo effects [43]. Supportive therapy [44] as a manualized, non-specific psychological intervention has been previously used in clinical trials [45]. It includes common factors such as elicitation of affect, treatment context, empathy, reflective listening, and feeling understood. It will be delivered in the same frequency and on the same occasions as SEPT (three individual sessions and three booster calls).

Sessions 1, 2 and 3: In the supportive therapy session contents may vary; there are no specific topics therapists need to address, there is no patient material and no homework. Nevertheless, every session can be structured into three phases: the beginning, the therapeutic dialog and the end. At the beginning patients are asked about relevant themes they want to talk about. During the therapeutic dialog patients lead the session and the therapist follows, while concentrating especially on the validation of patients' affects. It is suggested to talk about any topics that appear to have an affective valence to the patients. The therapist focuses on creating a warm atmosphere and shows empathy and unconditional positive regard towards the patient. At the ending of all sessions all themes are reviewed with the focus on the affect of the individual patient.

In the control group booster calls are conducted simultaneously to those in the intervention group, but they do not follow a particular structure. Patients are asked about their feelings since the last session.

\section{Measures}

Assessment occurs at four measurement points (see Table 2): at baseline approximately two weeks after surgery, after the intervention (post-intervention), and three and six months after the start of medication intake (follow-up). Questionnaires are applied by blinded research assistants at all measurement points. The following demographic and medical information will be obtained from medical charts and by baseline-interviews: socio-demographic status, age, BMI, health status, accompanying illnesses and medication (e.g., osteoporosis and medication), prior experiences with endocrine treatment (hormone contraception, hormone replacement therapy), stage of disease and tumor characteristics (UICC-stage, TNM-classification, Grade, ER/PR status, Her-2-status), type and course of primary treatment (breast-conserving surgery, mastectomy, radiotherapy, systemic treatment). Medical follow-up data include health and disease status, accompanying illnesses and medication for clinical outcome. All data will be validated and a plausibility check-up will be conducted.

\section{Primary outcomes}

Side effects will be measured with the General Assessment of Side Effects scale (GASE) $[46,47]$ which systematically assesses incidence and intensity of the 36 most common side effects. To assess the most frequent patient-reported adverse side effects from endocrine treatment the scale was modified by adding nine further symptoms (decreased interest in sex, weight gain, feeling of tension in breast, mood swings, abdominal bloating, vaginal dryness, bone fracture, pain during sexual intercourse, cataract) resulting in a total of 45 symptoms. Patients will be asked to rate the intensity of each listed bodily complaint during the past seven days on a four point Likert scale $(0=$ "complaint not present"; to 3 = "severe intensity"). Additionally, patients will indicate whether they attribute each of the present symptoms to current drug intake. It shows satisfactory psychometric properties [46].

\section{Secondary outcomes}

Quality of life will be assessed with the European Organization for Research and Treatment of Cancer Quality of Life Questionnaire (EORTC QLQ-C30) with breast module (QLQ-BR23) [48]. This questionnaire can be seen as the standard multidimensional instrument to measure quality of life among cancer patients. The QLQC30 consists of five functional scales, nine symptom scales and a global quality-of-life scale. The breast module is composed of eight breast cancer specific scales including four functional and four symptom scales. Both questionnaires have demonstrated good psychometric properties $[48,49]$.

Coping ability measures the perceived ability to manage occurring side effects. It will be accessed via a modified version of the GASE $[46,47]$ that will ask patients to rate their coping ability for each of the 45 listed side effects (i.e., "How good is your ability to manage the occurring adverse symptom?) on a Likert scale from $0=$ "good" to 4 = "bad".

Medication adherence will be measured with the German version of the Medication Adherence Report 
Table 2 Study measures

\begin{tabular}{|c|c|c|c|c|}
\hline & & $\begin{array}{c}\text { Baseline } \\
\text { pre-intervention }\end{array}$ & $\begin{array}{c}\text { Post- } \\
\text { intervention }\end{array}$ & $\begin{array}{c}\text { Follow-up ( } 3 \text { and } 6 \text { months after AET } \\
\text { intake) }\end{array}$ \\
\hline \multirow[t]{4}{*}{ Inclusion criteria } & Interview measures & & & \\
\hline & $\begin{array}{l}\text { Structured psychiatric interview } \\
\text { (mini-dips) }\end{array}$ & $x^{1}$ & & \\
\hline & Demographic and medical data & $x$ & & \\
\hline & Questionnaire measures & & & \\
\hline \multirow[t]{3}{*}{$\begin{array}{l}\text { Primary }+ \text { Secondary } \\
\text { Outcomes }\end{array}$} & $\begin{array}{l}\text { Physical symptoms and side } \\
\text { effects + coping (GASE+Coping) }\end{array}$ & $x$ & & $x$ \\
\hline & $\begin{array}{l}\text { Quality of life (EORTC QLQ C30 \& } \\
\text { BR23) }\end{array}$ & $x$ & & $x$ \\
\hline & $\begin{array}{l}\text { Adherence (MARS-D), Adherence } \\
\text { Intention }\end{array}$ & $x$ & $x$ & $x$ \\
\hline \multirow[t]{3}{*}{ Expectation Scales } & $\begin{array}{l}\text { Expected side effects }+ \text { expected } \\
\text { coping (GASE-Expect }+{ }^{*} \text { *Coping) }\end{array}$ & $x$ & $x$ & $x$ \\
\hline & Illness beliefs (IPQ-B) & $x$ & $x$ & $x$ \\
\hline & Beliefs about medicines (BMQ-D) & $x$ & $x$ & $x$ \\
\hline \multirow[t]{4}{*}{ Process Variables } & Fear of progression (PA-F-K) & $x$ & $x$ & $x$ \\
\hline & Anxiety and depression (HADS) & $x$ & $x$ & $x$ \\
\hline & Knowledge hormone receptor & $x$ & $x$ & $x$ \\
\hline & Treatment evaluation & & $x^{2}$ & \\
\hline \multirow[t]{4}{*}{ Control Variables } & $\begin{array}{l}\text { Somatosensory amplification } \\
\text { (SSAS) }\end{array}$ & $x$ & & \\
\hline & $\begin{array}{l}\text { Prior experiences with endocrine } \\
\text { treatment }\end{array}$ & $x$ & & \\
\hline & Partnership quality & $x$ & & \\
\hline & Additional medication & $x$ & & $x$ \\
\hline
\end{tabular}

Notes:

${ }^{1}$ The psychiatric interview is conducted prior to the measurement to check the inclusion criteria.

${ }^{2}$ Completed after every therapy session in the intervention groups only.

Scale (MARS) [50,51]. This scale gives an indication of the extent to which non-adherent behaviors occur, including how often patients have consciously not taken their medicines or forgotten to take them. It has been used to measure the adherence in endocrine treatment before [52]. Additionally, the adherence intention, the actual adherence and the attitude towards in cursive will be assessed with three single items.

\section{Expectations}

Side effect expectations refer to the patients' cognitive representations of the undesired effects related to a specific treatment. They will be measured with the General Assessment of Expected Side Effects Scale (GASE-expect), which is a modified version of the GASE-scale $[46,47]$ and was designed to measure patients' pharmacological response expectations with regard to the 45 most common side effects, including non-specific and specific complaints of endocrine therapy. Patients are instructed to indicate if and how strongly they expect to suffer from each potential side effect within the first three months of endocrine treatment.
Self-efficacy expectations about coping refer to the degree to which patients believe they are able to manage occurring side effects. They will be assessed using a modified version of the GASE-expect. Patients are asked to indicate their coping expectations for each of the 45 listed side effects (i.e., "Will I be able to manage occurring adverse symptoms?").

Treatment expectations: Expectations about medicines in general as well as specific concerns and necessity beliefs about endocrine therapy will be assessed with the German version of the Beliefs About Medicines Questionnaire (BMQ) [19,50]. The BMQ has previously been used to assess medications beliefs in breast cancer patients [52]. Additionally, pre-treatment expectations regarding SEPT and ACG will be assessed using single items.

Illness expectations: Expectations e.g., about time course, consequences, personal and treatment controllability of breast cancer, will be measured with the brief illness perception questionnaire B-IPQ [53]. Each single item represents a scale (in addition to the above mentioned: concerns, emotional response, coherence and aspects of identity). The cause-scale was excluded. This questionnaire 
has previously been used in cancer patients [54]. As suggested, the words "illness" and "treatment" were replaced with "breast cancer" and "endocrine therapy" (Table 2).

\section{Additional variables}

Fear of progression will be assessed with the short form of the of the Fear of Progression Questionnaire (PA-F-K) [55] consisting of 12 statements (e.g., being afraid of disease progression). It has shown good psychometric properties in breast cancer patients [56].

Anxiety and depression will be measured with the German Hospital Anxiety and Depression Scale (HADS-D) [38,39], which rates the severity of seven symptoms of anxiety and seven symptoms of depression over the past week and was designed for use in persons with physical illnesses. It has shown good psychometric properties in breast cancer patients [57].

Treatment evaluation will be measured in both intervention groups (ACG and SEPT). After every session, therapist and patient will rate their satisfaction with the unit using 12 items.

Knowledge about the patient's own tumor hormonereceptor status will be assessed by one item [58].

Somatosensory Amplification is the tendency to perceive ambiguous sensory events as unpleasant and will be assessed with the Somatosensory Amplification Scale (SSAS) [59]. The scale consists of 10 items (e.g., "I am often aware of various things happening within my body") and shows high validity and acceptable reliability in samples with breast cancer [60].

Partnership quality will be measured with one single item of the short form of the Partnership Questionnaire (PFB-K) [61] with reference to Terman [62] asking "How happy would you rate your partnership at the moment?", which has been recommended for assessing satisfaction with a partnership.

\section{Data analysis}

Missing values will be replaced using multivariate imputation techniques. A repeated measures multivariate analysis of variances (MANOVA) will be used to analyze treatment effects. To identify predictors of treatment outcome, multiple regression analyses will be conducted. To analyze pathways of the effects, mediator analyzes will be computed. Case studies will be performed to illustrate characteristics of the treatment processes from patients' und therapists' perspectives. Level of significance will be set at $\alpha=.05$.

\section{Baseline demographic data}

Preliminary baseline demographic data was assessed in $\mathrm{N}=55$ participating women with a primary breast cancer diagnosis. On average patients were 54.8 years old $(\mathrm{SD}=8.2$, range $=39-71$ years $)$, and mostly married or living with a partner (61.8\%). Other patients were single (12.7\%), widowed $(5.5 \%)$ or divorced (20.0\%). The majority of patients had primary education (63.6\%), other patients finished secondary education (16.4\%) or university education $(20.0 \%)$.

More than half of the patients were diagnosed with stage I breast cancer (69.1\%), further $27.2 \%$ of patients were diagnosed with stage II and additional 3.6\% with stage III. Most patients $(89.1 \%)$ received breast conserving therapy and only $10.9 \%$ mastectomy. A large group of patients (69.1\%) was scheduled to undergo first-line endocrine treatment with tamoxifen (+/- GnRH-analoga). A third generation aromatase inhibitor (i.e. anastrozole, letrozole or exemestan) was recommended to the other patients (30.9\%).

\section{Discussion}

Although expectations have been found to predict the occurrence of side effects in cancer patients, this study presents the first randomized controlled trial evaluating a short-term cognitive-behavioral intervention to prevent side effects during adjuvant endocrine therapy by optimizing breast cancer patients' expectations. The side-effect prevention training (SEPT) is compared with an attention control group (ACG) receiving supportive therapy and a standard medical care group (SMC) receiving standard treatment for breast cancer patients and additional oral and written information about adjuvant endocrine treatment. The primary outcomes are the occurrence of side effects three and six months after the start of intake of endocrine therapy. Further beneficial effects for quality of life, coping ability and adherence to medication are evaluated. Patients' response expectations, expectations about coping ability and expectations about treatment and illness are analyzed as mediators. The study also gives some insights into characteristics of patients who benefit the most from SEPT and of patients at high risk of developing side effects.

If SEPT is found to be effective, it could be integrated into daily clinical practice. Effects of preventing non-specific symtoms and nocebo side effects may improve the quality of life during treatment, lead to better medication adherence, and thereby may help to reduce progression and mortality in breast cancer patients and decrease costs of treatment. The training could be delivered into health care settings and applied by trained and supervised health care professionals. In addition, the study will provide insights into pathways of clinical nocebo effects and nonspecific side effects that may be applicable to other fields of illness and medication.

The study has some limitations that need consideration. First, our study design does not allow complete control regarding information patients may receive from their gynecologists about side effects of endocrine treatment, coping possibilities and about what happens while 
patients are in rehabilitation clinics. Another problem is that the study does not include patients who decide to start endocrine therapy before the baseline measurement. Presumably, patients in this group experience the strongest feelings of anxiety of progression and have more negative illness beliefs. This study does not provide conclusions about the efficacy of single treatment elements or differential indications. If treatment effects are robust, future studies are needed to analyze the particular influences of those factors.

The optimization of expectations might be a promising pathway to improve patients' quality of life during medication intake. So far, this is the first study investigating a psychological prevention program for side effects with the explicit focus on patients' expectations. The results will provide implications for a possible integration of evidence-based prevention training into clinical practice.

\author{
Abbreviations \\ ACG: Attention control group; AET: Adjuvant endocrine therapy; \\ ER/PR: Estrogen receptor/ progesterone receptor; $B C T$ : Breast-conserving \\ therapy; GnRH: Gonadotropin-releasing hormone; SEPT: Side-effect \\ prevention training; SMC: Standard medical care.
}

\section{Competing interests}

The authors declare that they have no conflict of interests that could have influenced the content of this report. WR received honoraria from Astra Zeneca, Heel, and Berlin Chemie for consultation and talks about placebo effects and medication adherence. YN received honoraria from Berlin Chemie and Atlantis Healthcare for consultation and talks about placebo effects and medication adherence.

\section{Authors' contributions}

$\mathrm{PvB}$ is a PhD student in the project, participated in writing the intervention manual, carries out the psychological interventions and drafted the manuscript. FS is also a PhD student, contributed to the intervention manual and carries out the psychological interventions. USA and WR are co-investigators and participated in the conception of the study. YN is the principal investigator, formulated the research questions, conceptualized the study design and wrote the intervention manual. All authors approved the final version of the manuscript.

\section{Acknowledgements}

The authors thank: Miriam Bartek for contributing to the patient materials; Nora Kaestle and Tobias Weber for assisting in the patient recruitment and data management; Prof. Dr. Arthur Barsky (Harvard Medical School) for his support in developing the research design; Dr. Sabine Rehahn-Sommer (Marburg, Germany) for her ongoing supervision of the psychological interventions and Dr. Meike Shedden Mora for her helpful comments on the manuscript. The authors further thank the team of the department of Gynecology, Gynecological Endocrinology and Oncology, Philipps University, Marburg, Germany, for their help with the patient recruitment.

\section{Sponsors}

The study is sponsored by a grant of the German Research Foundation (DFG) to Dr. Nestoriuc (NE 1635/2-1) and is a subproject of the DFG research unit (FOR 1328): Expectation and conditioning as basic processes of the placebo and nocebo response: From neurobiology to clinical applications.

\section{Author details}

${ }^{1}$ Department of Clinical Psychology and Psychotherapy, Philipps University, 35032, Marburg, Germany. ${ }^{2}$ Department of Gynecology, Gynecological Endocrinology and Oncology, Breast Cancer Center, Philipps University, Marburg, Germany. 'Department of Clinical Psychology, Hamburg University, Hamburg, Germany.
Received: 8 May 2013 Accepted: 12 September 2013

Published: 18 September 2013

\section{References}

1. Burstein HJ, Prestrud AA, Seidenfeld J, Anderson H, Buchholz TA, Davidson NE, Gelmon KE, Giordano SH, Hudis CA, Malin J, et al: American society of clinical oncology clinical practice guideline: update on adjuvant endocrine therapy for women with hormone receptor-positive breast cancer. J Clin Oncol 2010, 28:3784-3796.

2. Kreienberg R, Albert US, Follmann M, Kopp I, Kühn T, Wöckel A, Zemmler T: Interdisziplinäre S3-Leitlinie für die Diagnostik, Therapie und Nachsorge des Mammakarzinoms. In Leitlinienprogramm Onkologie. Edited by AWMF, Deutsche Krebsgesellschaft e.V. und Deutsche Krebshilfe e.V. Zuckschwerdt: München; 2012.

3. Dowsett M, Cuzick J, Ingle J, Coates A, Forbes J, Bliss J, Buyse M, Baum M, Buzdar A, Colleoni M, et al: Meta-analysis of breast cancer outcomes in adjuvant trials of aromatase inhibitors versus tamoxifen. $J$ Clin Oncol 2010, 28:509-518.

4. Howell A, Cuzick J, Baum A, Buzdar A, Dowsett M, Forbes J, Hoctin-Boes G, Houghton J, Locker GY, Tobias S: Results of the ATAC (Arimidex, Tamoxifen, Alone or in Combination) trial after completion of 5 years adjuvant treatment for breast cancer. Lancet 2005, 365:60-62.

5. Ziller V, Kalder M, Albert US, Holzhauer W, Ziller M, Wagner U, Hadji P: Adherence to adjuvant endocrine therapy in postmenopausal women with breast cancer. Ann Oncol 2009, 20:431-436.

6. Neugut A, Hillyer G, Kushi L, Lamerato L, Leoce N, Nathanson S, Ambrosone C, Bovbjerg D, Mandelblatt J, Magai C, et al: Non-initiation of adjuvant hormonal therapy in women with hormone receptor-positive breast cancer: the breast cancer quality of care study (BQUAL). Breast Cancer Res Treat 2012, 134:419-428.

7. Hershman DL, Kushi LH, Shao T, Buono D, Kershenbaum A, Tsai W-Y, Fehrenbacher L, Lin Gomez S, Miles S, Neugut Al: Early discontinuation and nonadherence to adjuvant hormonal therapy in a cohort of 8,769 early-stage breast cancer patients. J Clin Oncol 2010, 28:4120-4128.

8. Partridge AH, LaFountain A, Mayer E, Taylor BS, Winer E, Asnis-Alibozek A: Adherence to initial adjuvant anastrozole therapy among women with early-stage breast cancer. J Clin Oncol 2008, 26:556-562.

9. Hershman D, Shao T, Kushi L, Buono D, Tsai W, Fehrenbacher L, Kwan M, Gomez S, Neugut A: Early discontinuation and non-adherence to adjuvant hormonal therapy are associated with increased mortality in women with breast cancer. Breast Cancer Res Treat 2011, 126:529-537.

10. Demissie S, Silliman RA, Lash TL: Adjuvant tamoxifen: predictors of use, side effects, and discontinuation in older women. J Clin Oncol 2001, 19:322-328.

11. Cella D, Fallowfield L, Barker P, Cuzick J, Locker G, Howell A: Quality of life of postmenopausal women in the ATAC ("Arimidex", Tamoxifen, Alone or in Combination) trial after completion of 5 years' adjuvant treatment for early breast cancer. Breast Cancer Res Treat 2006, 100:273-284.

12. Cella D, Fallowfield L: Recognition and management of treatment-related side effects for breast cancer patients receiving adjuvant endocrine therapy. Breast Cancer Res Treat 2008, 107:167-180.

13. Gibson L, Lawrence D, Dawson C, Bliss J: Aromatase inhibitors for treatment of advanced breast cancer in postmenopausal women. Cochrane Database Syst Rev 2009, 4, CD003370.

14. Barsky AJ, Saintfort R, Rogers MP, Borus JF: Nonspecific medication side effects and the nocebo phenomenon. JAMA 2002, 287:622-627.

15. Rief W, Avorn J, Barsky AJ: Medication-attributed adverse effects in placebo groups: implications for assessment of adverse effects. Arch Intern Med 2006, 166:155-160.

16. Whelan TJ, Goss PE, Ingle JN, Pater JL, Tu D, Pritchard K, Liu S, Shepherd LE, Palmer M, Robert NJ, et al: Assessment of quality of life in MA.17: a randomized, placebo-controlled trial of letrozole after 5 years of tamoxifen in postmenopausal women. J Clin Oncol 2005, 23:6931-6940.

17. Colloca L, Miller FG: Role of expectations in health. Curr Opin Psychiatr 2011, 24:149.

18. Rief W, Bingel U, Schedlowski M, Enck P: Mechanisms involved in placebo and nocebo responses and implications for drug trials. Clin Pharmacol Ther 2011, 90:722-726.

19. Nestoriuc Y, Orav EJ, Liang MH, Horne R, Barsky AJ: Predection of nonspecific side effects in rheumatoid arthritis patients by beliefs about medicines. Arthritis Care Res 2010, 62:791-799.

20. Roscoe JA, Jean-Pierre P, Shelke AR, Kaufman ME, Bole C, Morrow GR: The role of patients' response expectancies in side effect development and control. Curr Probl Cancer 2006, 30:40-98. 
21. Colagiuri B, Zachariae R: Patient expectancy and post-chemotherapy nausea: a meta-analysis. Ann Behav Med 2010, 40:3-14.

22. Sohl SJ, Schnur JB, Montgomery GH: A meta-analysis of the relationship between response expectancies and cancer treatment-related side effects. J Pain Symptom Manage 2009, 38:775-784.

23. Nestoriuc Y, Schuricht F, von Blanckenburg P, Albert US, Rief W: Der Einfluss von Erwartungen auf den Behandlungsverlauf der Antihormonellen Therapie bei Brustkrebs-Patientinnen [abstract]. Verhaltenstherapie 2011, 21(Suppl 1):17

24. Nestoriuc $Y$, Schuricht $F$, von Blanckenburg $P$, Rief W, Albert US: Communicating side effects by informing about possible benefits and harms: effects on breast cancer patients' satisfaction, knowledge and expectations [abstract]. J Cancer Res Clin Oncol 2012, 138(Suppl 1):77.

25. Rozema $H$, Völlink $T$, Lechner $L$ : The role of illness representations in coping and health of patients treated for breast cancer. Psycho-Oncol 2009, 18:849-857.

26. Leventhal H, Nerenz D, Steele DJ: Illness representations and coping with health threats. In Handbook of psychology and health volume IV social psychology aspects of health. Edited by Baum A, Taylor SE, Singer JE. Hillsdale, New Jersey: Erlbaum; 1984:219-252.

27. McGinty HL, Goldenberg JL, Jacobsen PB: Relationship of threat appraisal with coping appraisal to fear of cancer recurrence in breast cancer survivors. Psycho-Oncol 2012, 21:203-210.

28. Benedetti F, Lanotte M, Lopiano L, Colloca L: When words are painful: unraveling the mechanisms of the nocebo effect. Neuroscience 2007, 147:260-271.

29. Liu L, Fiorentino L, Natarajan L, Parker BA, Mills PJ, Sadler GR, Dimsdale JE, Rissling M, He F, Ancoli-Israel S: Pre-treatment symptom cluster in breast cancer patients is associated with worse sleep, fatigue and depression during chemotherapy. Psycho-Oncol 2009, 18:187-194.

30. de la Cruz M, Hui D, Parsons HA, Bruera E: Placebo and nocebo effects in randomized double-blind clinical trials of agents for the therapy for fatigue in patients with advanced cancer. Cancer 2010, 116:766-774.

31. Fischer MJ, Wiesenhaan ME, Heijer AD-d, Kleijn WC, Nortier JWR, Kaptein AA: From despair to hope: a longitudinal study of illness perceptions and coping in a psycho-educational group intervention for women with breast cancer. Br J Health Psychol 2012. DOl:10.1111/j.2044-8287.2012.02100.x.

32. Roscoe JA, O'Neill M, Jean-Pierre P, Heckler CE, Kaptchuk TJ, Bushunow P, Shayne M, Huston A, Qazi R, Smith B: An exploratory study on the effects of an expectancy manipulation on chemotherapy-related nausea. $J$ Pain Symptom Manage 2010, 40:379-390.

33. Colloca L, Miller FG: The nocebo effect and its relevance for clinical practice. Psychosom Med 2011, 73:598-603.

34. Duijts SFA, van Beurden M, Oldenburg HSA, Hunter MS, Kieffer JM, Stuiver MM, Gerritsma MA, Menke-Pluymers MBE, Plaisier PW, Rijna $\mathrm{H}$, et al: Efficacy of cognitive behavioral therapy and physical exercise in alleviating treatment-induced menopausal symptoms in patients with breast cancer: results of a randomized, controlled, multicenter trial. I Clin Oncol 2012, 30:4124-4133.

35. Mann E, Smith MJ, Hellier J, Balabanovic JA, Hamed H, Grunfeld EA, Hunter MS: Cognitive behavioural treatment for women who have menopausal symptoms after breast cancer treatment (MENOS 1): a randomised controlled trial. Lancet Oncol 2012, 13:309-318.

36. Margraf J: Diagnostisches Kurz-Interview bei psychischen Störungen (Mini-DIPS). Göttingen: Hogrefe; 1994

37. Faul F, Erdfelder E, Lang A-G, Buchner A: G*power 3: a flexible statistical power analysis program for the social, behavioral, and biomedical sciences. Behav Res Methods 2007, 39:175-191.

38. Zigmond AS, Snaith RP: The hospital anxiety and depression scale. Acta Psychiatr Scand 1983, 67:361-370.

39. Herrmann C, Buss U, Snaith RR: Hospital anxiety and depression scale Deutsche Version (HADS-D): manual. Bern: Huber; 1995.

40. Abramson J: WINPEPI updated: computer programs for epidemiologists, and their teaching potential. Epidemiol Perspect Innov 2011, 8:1.

41. McGurk R, Fallowfield L, Winters Z: Information provision for patients by breast cancer teams about the side-effects of hormone treatments. Eur J Cancer 2006, 42:1760-1767.

42. Monnier A: Clinical management of adverse events in adjuvant therapy for hormone-responsive early breast cancer. Ann Oncol 2007, 18:36-44.

43. Hautzinger M: Die Plazebokontrollgruppe in der Psychotherapieforschung. Psychotherapie 2001, 6:199-204.
44. Markowitz JC, Manber R, Rosen P: Therapists' responses to training in brief supportive psychotherapy. Am J Psychother 2008, 62:67-81.

45. Cohen L, Parker PA, Vence L, Savary C, Kentor D, Pettaway C, Babaian R, Pisters L, Miles B, Wei $Q$, et al: Presurgical stress management improves postoperative immune function in men with prostate cancer undergoing radical prostatectomy. Psychosom Med 2011, 73:218-225.

46. Rief W, Barsky AJ, Glombiewski JA, Nestoriuc Y, Glaesmer H, Braehler E: Assessing general side effects in clinical trials: reference data from the general population. Pharmacoepidemiol Drug Saf 2011, 20:405-415.

47. Rief W, Glombiewski JA, Barsky AJ: General assessment of side effects: GASE. Bern: Huber (electronic version); 2009

48. Aaronson NK, Ahmedzai S, Bergman B, Bullinger M, Cull A, Duez NJ, Filiberti A, Flechtner $\mathrm{H}$, Fleishman SB, de Haes JC: The European organization for research and treatment of cancer QLQ-C30: a quality of life instrument for use in international clinical trials in oncology. J Natl Canc Inst 1993, 85:365-376.

49. Sprangers MA, Groenvold M, Arraras Jl, Franklin J, te Velde A, Muller M, Franzini L, Williams A, de Haes HC, Hopwood P, et al: The European organization for research and treatment of cancer breast cancer-specific quality-of-life questionnaire module: first results from a three-country field study. J Clin Oncol 1996, 14:2756-2768.

50. Horne R, Weinman J, Hankins M: The beliefs about medicines questionnaire: the development and evaluation of a new method for assessing the cognitive representation of medication. Psychol Health 1999, 14:1.

51. Mahler C, Hermann K, Horne R, Ludt S, Haefeli WE, Szecsenyi J, Jank S: Assessing reported adherence to pharmacological treatment recommendations. translation and evaluation of the medication adherence report scale (MARS) in Germany. J Eval Clin Pract 2010, 16:574-579.

52. Grunfeld EA, Hunter MS, Sikka P, Mittal S: Adherence beliefs among breast cancer patients taking tamoxifen. Patient Educ Couns 2005, 59:97-102.

53. Broadbent E, Petrie KJ, Main J, Weinman J: The brief illness perception questionnaire. J Psychosom Res 2006, 60:631-637.

54. Grunfeld EA, Low E, Cooper AF: Cancer survivors' and employers' perceptions of working following cancer treatment. Occup Med 2010, 60:611-617.

55. Mehnert A, Herschbach P, Berg P, Henrich G, Koch U: Progredienzangst bei Brustkrebspatientinnen-Validierung der Kurzform des Progredienzangstfragebogens PA-F-KF. Z Psychosom Med Psychother 2006, 52:274-288

56. Mehnert A, Berg P, Henrich G, Herschbach P: Fear of cancer progression and cancer-related intrusive cognitions in breast cancer survivors. Psycho-Oncol 2009, 18:1273-1280.

57. Alexander S, Palmer C, Stone P: Evaluation of screening instruments for depression and anxiety in breast cancer survivors. Breast Cancer Res Treat 2010, 122:573-578

58. Albert US, Zemlin C, Hadji P, Ziller V, Kuhler B, Frank-Hahn B, Wagner U, Kalder M: The impact of breast care nurses on patients' satisfaction, understanding of the disease, and adherence to adjuvant endocrine therapy. Breast Care 2011, 6(3):221-226.

59. Barsky AJ, Goodson JD, Lane RS, Cleary PD: The amplification of somatic symptoms. Psychosom Med 1988, 50:510-519.

60. Gurevich M, Devins GM, Wilson C, McCready D, Marmar CR, Rodin GM: Stress response syndromes in women undergoing mammography: a comparison of women with and without a history of breast cancer. Psychosom Med 2004, 66:104-112.

61. Kliem S, Job A-K, Kröger C, Bodenmann G, Stöbel-Richter Y, Hahlweg K, Brähler E: Entwicklung und Normierung einer Kurzform des Partnerschaftsfragebogens (PFB-K) an einer repräsentativen deutschen Stichprobe. Z Klin Psychol Psychother 2012, 41:81-89.

62. Terman LM, Butterwieser P, Ferguson LW, Johnson WB, Wilson DP: Psychological factors in marital happiness. New York: McGraw-Hill; 1983.

\section{doi:10.1186/1471-2407-13-426}

Cite this article as: von Blanckenburg et al:: Optimizing expectations to prevent side effects and enhance quality of life in breast cancer patients undergoing endocrine therapy: study protocol of a randomized controlled trial. BMC Cancer 2013 13:426. 